\title{
AVALIAÇÃO DA DINÂMICA LATERAL E DOS MECANISMOS DE TRANSPORTE NO ESTUÁRIO DO RIO CAMBORIÚ
}

\author{
FRANKLIN-SILVA, L. \& C.A.F. SCHETTINI \\ lelo@cttmar.univali.br \\ Centro de Ciências Tecnológicas da Terra e do Mar/Universidade do Vale do Itajaí \\ (CTTMar/UNIVALI)
}

\begin{abstract}
RESUMO
O principal objetivo deste trabalho é avaliar a estrutura hidrodinâmica lateral do estuário do Rio Camboriú, em períodos de maré de quadratura e sizígia, bem como definir os principais mecanismos de transportes atuantes neste estuário. Nas duas campanhas realizadas, os dados de velocidade de corrente e salinidade foram adquiridos a cada hora durante dois ciclos completos de maré ( 25 horas). Os perfis verticais das variáveis foram obtidos em três estações na seção transversal do estuário. Nas condições estudadas, durante a maré de quadratura este estuário foi classificado como parcialmente estratificado (Tipo $2 b$ ) e durante maré de sizígia como verticalmente homogêneo (Tipo 1a). Características morfológicas associadas à presença de uma onda estacionária (Seiche), provavelmente originada na enseada de Balneário Camboriú, promovem uma diferenciação nos perfis de velocidade e direção de corrente entre as estações amostrais durante maré de quadratura. Para a mesma situação observa-se uma estratificação da coluna de água, ocorrendo a predominância de processos advectivos devido à descarga fluvial. Durante o período de maré de sizígia, o efeito da amplitude de maré associado à baixa profundidade do sistema, proporciona uma coluna de água homogênea, ocorrendo a predominância de processos de difusão turbulenta.
\end{abstract}

Palavras Chave: hidrodinâmica, transporte, estuário, Rio Camboriú.

\section{ASSESSMENT OF THE LATERAL DYNAMICS AND THE MECHANISMS OF TRANSPORT IN THE ESTUARY OF CAMBORIÚ RIVER}

\begin{abstract}
The main objective of this work is evaluate the structure lateral hydrodynamics of the estuary of the Camboriú River, in periods of neap and spring tide, as well as defining the main mechanisms of operating transports in this estuary. In the two carried campaigns, data of speed current and salinity had been acquired to each hour during two complete tide's cycles ( 25 hours). The vertical profiles had been gotten in three stations in the transversal section of the estuary. In the conditions studied, during neap tide this estuary is classified as partially stratified (Type 2b) and vertically homogeneous during spring tide (Type 1a). Morphologic characteristics associates to the presence of a standing wave (Seiche), probably originated in the Camboriú bay, promote a differentiation in the current profiles between the stations during neap tide. For the same situation a stratification of the water column is observed, occurring the predominance of advective processes due to fluvial discharge. During spring tide, the effect of the amplitude of tide associated with low depth of the system provides a homogeneous water column, occurring the predominance of processes of turbulent diffusion.
\end{abstract}

Key words: hydrodynamics, transport, estuary, Camboriú River. 


\section{INTRODUÇÃO}

A qualidade ambiental de um estuário está diretamente relacionada com as características hidrodinâmicas presentes, ou seja, com a capacidade de renovação de suas águas. A forma pela qual ocorre a mistura das águas é um elemento chave para caracterizar se o sistema apresenta características de retenção ou de exportação de sedimentos e outros constituintes dissolvidos e particulados. A conseqüência da retenção de materiais é o efeito acumulativo de poluentes nos sedimentos de fundo (Hunkins, 1981; Shubel \& Carter, 1984).

Os lançamentos de esgotos ocasionados pela falta de planejamento ou infraestrutura das cidades localizadas no entorno dos estuários, representam a principal causa dos impactos ambientais presentes nestes sistemas. Segundo Miranda et. al. (2002), a descarga de efluentes sanitários das comunidades adjacentes, embarcações e zonas portuárias, além de provocar a contaminação da água por bactérias e vírus, resulta no aumento do consumo de oxigênio, contribuindo com maior deposição de materiais, dando origem a um lodo anaeróbico nas regiões costeiras. Em conseqüência dessas descargas, as áreas afetadas passam a sofrer grandes oscilações no teor de oxigênio dissolvido, prejudicando a biota em geral.

A cidade de Balneário Camboriú, localizada às margens do estuário do Rio Camboriú, é um importante centro turístico do sul do Brasil. Embora exista tratamento de esgoto no município, uma grande parte é lançada em fossas sépticas ou diretamente nos cursos de água, que desembocam no estuário. Com o estabelecimento dos padrões de circulação, é possível avaliar os mecanismos de mistura deste sistema.

Assim, o principal objetivo desta pesquisa é avaliar a estrutura lateral do estuário do rio Camboriú, em períodos de maré de quadratura e sizígia, bem como definir os principais mecanismos de transportes atuantes neste estuário.

\section{Área de Estudo}

A bacia hidrográfica do rio Camboriú apresenta uma área de $200 \mathrm{Km}^{2}$, compreendendo apenas dois municípios. A montante está localizada a cidade de Camboriú, que apresenta uma economia baseada na agricultura e na extração mineral. A jusante localiza-se Balneário Camboriú, uma das maiores cidades da região Sul durante os meses de verão, quando atinge uma população de cerca de 1.000 .000 habitantes, excedendo quase 10 vezes a sua população normal (Polette, 1999). Na área estuarina deságua o rio, onde se concentram ilhas fluviais que exibem uma vegetação característica de mangues. O rio Camboriú possui cerca de 40 $\mathrm{Km}$ de extensão e deságua no extremo sul da praia de Balneário Camboriú, com uma largura aproximada de 120 metros.

Um dos principais problemas relacionados com a qualidade de vida e turismo local é o lançamento excessivo de efluentes domésticos neste estuário. Estudos realizados por Silva et al. (1999) indicaram presença de toxicidade em níveis significativos nos efluentes provenientes da estação de tratamento de esgotos.

De acordo com Pereira Filho et. al. (2001), este sistema é a principal via de acesso de nutrientes para a enseada de Balneário Camboriú. A principal forma de Nitrogênio Inorgânico Dissolvido é Amônio, com uma razão Nitrogênio:Fósforo elevada (45). A origem destes índices pode estar relacionada à entrada de esgotos clandestinos, efluentes agrícolas ou da estação de tratamento de esgotos. Segundo Siegle et al. (1997) a desembocadura do rio Camboriú apresentou nas últimas décadas alterações devido à influência antrópica, como: a diminuição do aporte de água doce ao estuário e 0 aterro dos mangues nas margens do rio, fatores estes que podem levar à redução de sua profundidade.

Resultados publicados em Schettini et al. (1996), classificaram este estuário como um tipo intermediário, entre altamente estratificado 
e parcialmente estratificado, de acordo com o esquema de Hansen \& Rattray (1966). Esta classificação foi baseada na estimativa de uma contribuição fluvial de $6 \mathrm{~m}^{3} \cdot \mathrm{s}^{-1}$. Atualmente estima-se que esta contribuição é aproximadamente a metade desta (FranklinSilva \& Schettini, 1997).

Baseando-se em informações de salinidade e velocidade de corrente em três níveis e num único ponto amostral, Siegle (1999) classificou o estuário como parcialmente estratificado (Tipo 2b). Neste experimento, os principais mecanismos de transporte encontrados foram devido a advecção fluvial e cisalhamento vertical, seguidos pelo efeito da turbulência.

\section{MATERIAIS E MÉTODOS}

\section{Coleta dos dados}

Foram realizadas duas campanhas para levantamentos de dados oceanográficos no estuário do Rio Camboriú, sendo uma sob condição de maré de quadratura (9 e 10 de abril de 1999) e a outra sob condição de maré de sizígia ( 27 e 28 de agosto de 1999). As estações de coleta localizaram-se próximas da desembocadura do estuário, estabelecendo uma seção transversal retilínea bem definida entre a ilha e o cais (Figura 1).

Os valores de salinidade, velocidade e direção de corrente foram coletados a cada hora durante dois ciclos completos de maré ( 25 horas). Os perfis foram obtidos nas três estações ao longo da seção transversal do estuário, utilizando-se de um correntógrafo marca Falmouth TM modelo 3D-ACM WAVE $®$ em modo de aquisição em tempo real. A taxa de aquisição do correntógrafo foi de 0,2 segundos, onde o equipamento foi disposto por um período de 10 segundos em patamares a cada $30 \mathrm{~cm}$ de coluna d'água. Ao lado do trapiche (Estação cais) foi instalado um marégrafo de pressão marca Richard Brancker TM modelo XL-205®, onde foram coletadas medidas de nível d'água a cada 30 minutos durante o experimento.

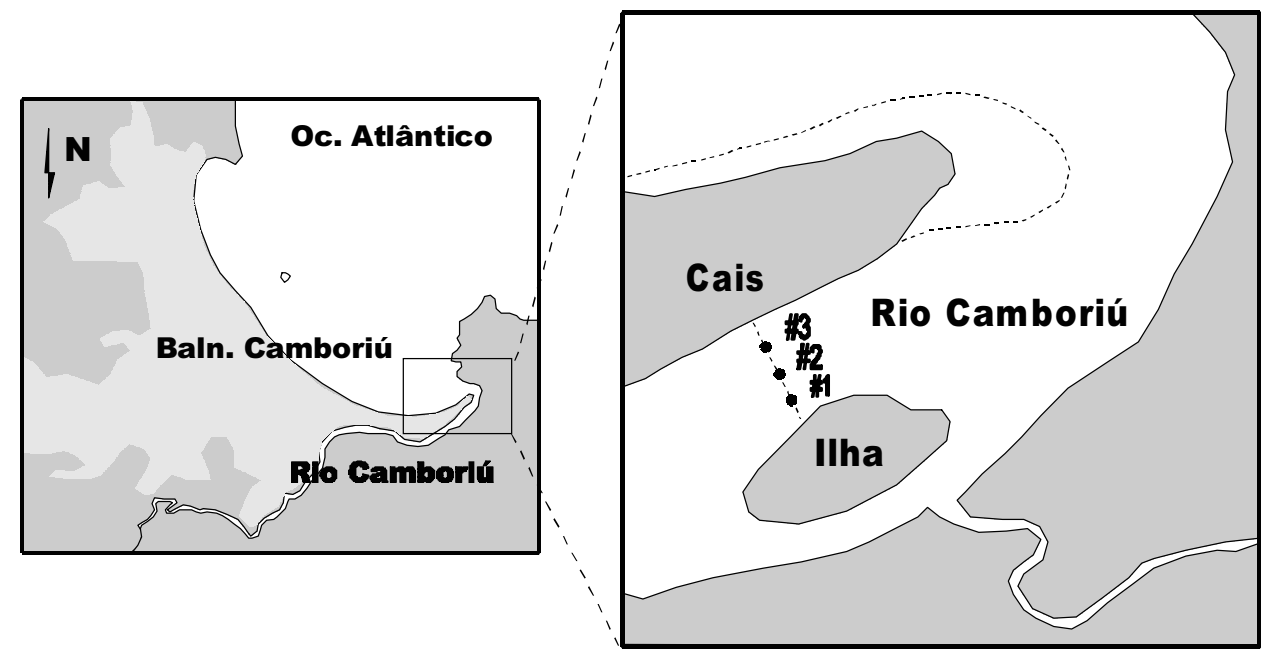

Figura 1 - Localização da área de estudo com as estações amostrais (\#) ao longo da seção transversal ( - ) do estuário do rio Camboriú. 
Através da relação entre os dados diários de vazão do Rio Itajaí-Açú e a área da bacia de drenagem a montante do local de coleta destes dados $\left(11.110 \mathrm{~km}^{2}\right)$, foi possível estimar os valores de vazão do Rio Camboriú para as condições de maré estudadas. Esta estimativa foi realizada devido à falta de dados de vazão na área estudada. É importante salientar que as bacias hidrográficas em questão, apesar de diferentes no tamanho, são adjacentes e estão sob o mesmo regime meteorológico. Durante a maré de quadratura e sizígia foi estimada uma vazão de $3,8 \mathrm{~m}^{3} / \mathrm{s}$ e $2,1 \mathrm{~m}^{3} / \mathrm{s}$, respectivamente. Os valores de vazão encontrados corroboram com Franklin-Silva (1997).

\section{Tratamento e Análise dos dados}

Os dados de velocidade de corrente foram decompostos em vetores $u$ e $v$, filtrados por um filtro de três pontos $(1 / 4,1 / 2,1 / 4)$ recursivo que elimina oscilações de alta freqüência e recompostos novamente para coordenadas polares. Os valores de direção de corrente foram analisados através de histogramas, definindo a direção na qual ocorre a inversão dos valores de velocidade de corrente de enchente e vazante, convencionando valor negativo para correntes com direção ao oceano (saindo) e valores positivos para correntes com direção ao continente (entrando).

Em função da diferença de níveis amostrados em cada perfil vertical ao longo do tempo e lateralmente, os dados foram reduzidos para profundidade adimensional, interpolados linearmente, e reamostrados em cinco níveis. Desta forma, todos os perfis apresentam o mesmo número de amostras.

A classificação do estuário foi realizada segundo Hansen \& Rattray (1966), através da definição dos parâmetros estratificação e circulação, que representam os eixos ortogonais ordenada e abscissa do diagrama. O parâmetro estratificação é obtido através da divisão entre a diferença da salinidade do fundo e da superfície com salinidade média da coluna de água. O parâmetro circulação é definido pela divisão entre o valor da corrente residual na superfície e a velocidade da corrente gerada pela descarga de água doce.

Os mecanismos de transporte no estuário do Rio Camboriú foram determinados segundo a abordagem de Kjerfve (1986). De maneira geral, o fluxo de sal $(F)$ por unidade de largura de seção transversal pode ser calculado para cada nível de cada estação como

$$
\mathrm{F}=\int_{0}^{\mathrm{P}} \mathrm{uSdz}
$$

onde u é a velocidade instantânea normal à seção transversal, $S$ é a salinidade e $\mathrm{P}$ é a profundidade representativa de cada amostra da seção. Considerando que tanto a velocidade quanto a salinidade podem ser expressas como variações em torno de um valor médio temporal e espacial na forma de

$$
\begin{array}{r}
\mathrm{u}=\overline{\mathrm{u}}+<\overline{\mathrm{u}}>+\mathrm{u}^{\prime} \\
\& \\
\mathrm{~S}=\overline{\mathrm{S}}+<\overline{\mathrm{S}}>+\mathrm{S}^{\prime}
\end{array}
$$

onde os valores dentro de $<>$ representam médias temporais sobre ciclos de maré, a barra representa médias espaciais verticais e a apóstrofe representa o desvio em relação à média vertical. Substituindo u e $S$ da Eq. (4) pelas representações expressas pelas Eqs. (5), e integrando no tempo os resultados, poderemos expressar o termo do lado esquerdo da igualdade da Eq. (2) como

$$
<\mathrm{F}>=<\mathrm{P} \overline{\mathrm{uS}}>
$$

e o lado direito poderá se rescrito como 
$<F>=<P>\cdot\left\langle\bar{u}>\cdot\left\langle\bar{S}>+<P u^{\prime} S^{\prime}>+<\bar{u}>\right.\right.$. . $\left\langle P S^{\prime}>+<\bar{S}>\cdot<P u^{\prime}>+<P u^{\prime} S^{\prime}>+<P \overline{u^{\prime} S^{\prime}}>\right.$

$\mathrm{O} 1^{\circ}$ termo do lado direito da Eq. 7 representa o transporte advectivo devido à descarga fluvial, o $2^{\circ}, 3^{\circ}$ e $4^{\circ}$ estão associados a efeitos da maré, o $5^{\circ}$ representa o cisalhamento vertical e o $6^{\circ}$ representa a turbulência.

\section{RESULTADOS}

Durante a campanha de maré de quadratura, o nível de água apresentou uma variação máxima de $0,65 \mathrm{~m}$, sendo que o nível mínimo foi de -0,42 m e o máximo de 0,23 $\mathrm{m}$. Durante a campanha de maré de sizígia a máxima variação foi de $1,27 \mathrm{~m}$, sendo que o nível mínimo foi de $-0,65 \mathrm{~m}$ e o máximo de $0,62 \mathrm{~m}$ (Figura 2).

Durante o período de maré de quadratura foi observada uma dominância das correntes de maré vazante. Neste período o pico de velocidade instantânea de maré enchente foi cerca de $33 \mathrm{~cm} . \mathrm{s}^{-1}$ e de maré vazante cerca de $36 \mathrm{~cm} . \mathrm{s}^{-1}$ (Figura 3a). Para o período de maré de sizígia ocorreu um predomínio de correntes de maré enchente, sendo que o pico de velocidade instantânea de maré enchente foi cerca de $66 \mathrm{~cm} . \mathrm{s}^{-1}$ e de maré vazante cerca de
$86 \mathrm{~cm} . \mathrm{s}^{-1}$ (Figura 4a). Os valores negativos representam a saída de água do estuário.

A distribuição da salinidade apresentou padrões diferentes entre os tipos de marés amostradas. Durante a maré de quadratura a coluna de água permaneceu estratificada na maior parte do tempo, sendo observado uma pequena mistura em torno da décima terceira e vigésima quarta hora do experimento (Figura $3 b)$. Para a maré de sizígia a coluna d'água se apresentou verticalmente homogênea, sendo que ocorreu o predomínio de água marinha na seção transversal (Figura 4b). Nas duas situações do experimento não foram observadas diferenças significativas na distribuição vertical da salinidade entre as estações amostrais.

Comparando os valores de salinidade em cada ponto amostral nos períodos estudados, observa-se que este sistema apresenta uma variabilidade lateral de salinidade pequena. Segundo Franklin-Silva et. al. (1998) este padrão pode ser esperado, já que este sistema apresenta uma alta razão entre a altura da maré $(1,2 \mathrm{~m})$ e a profundidade média da seção transversal $(2,14 \mathrm{~m})$, bem como uma extensão lateral de $75 \mathrm{~m}$.

As Figuras 5 e 6 apresentam as médias temporais de velocidade de corrente e salinidade para condições de marés estudadas.

Através dos resultados obtidos, pode-se afirmar que ocorreu uma variação na

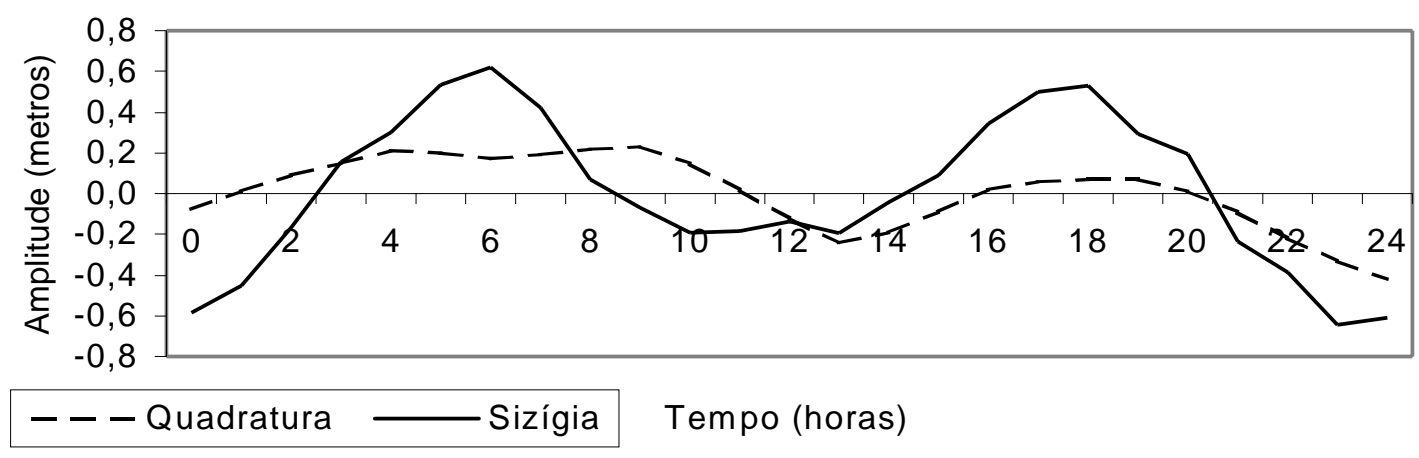

Figura 2 - Representação gráfica da maré durante condições de maré de quadratura e sizígia. 
classificação do estuário do rio Camboriú, segundo Hansen \& Rattray (1996). Em períodos de maré de quadratura este sistema é classificado como Tipo $2 \mathrm{~b}$ e durante o período de maré de sizígia como Tipo 1a (Figura 7). Os valores dos parâmetros estratificação e

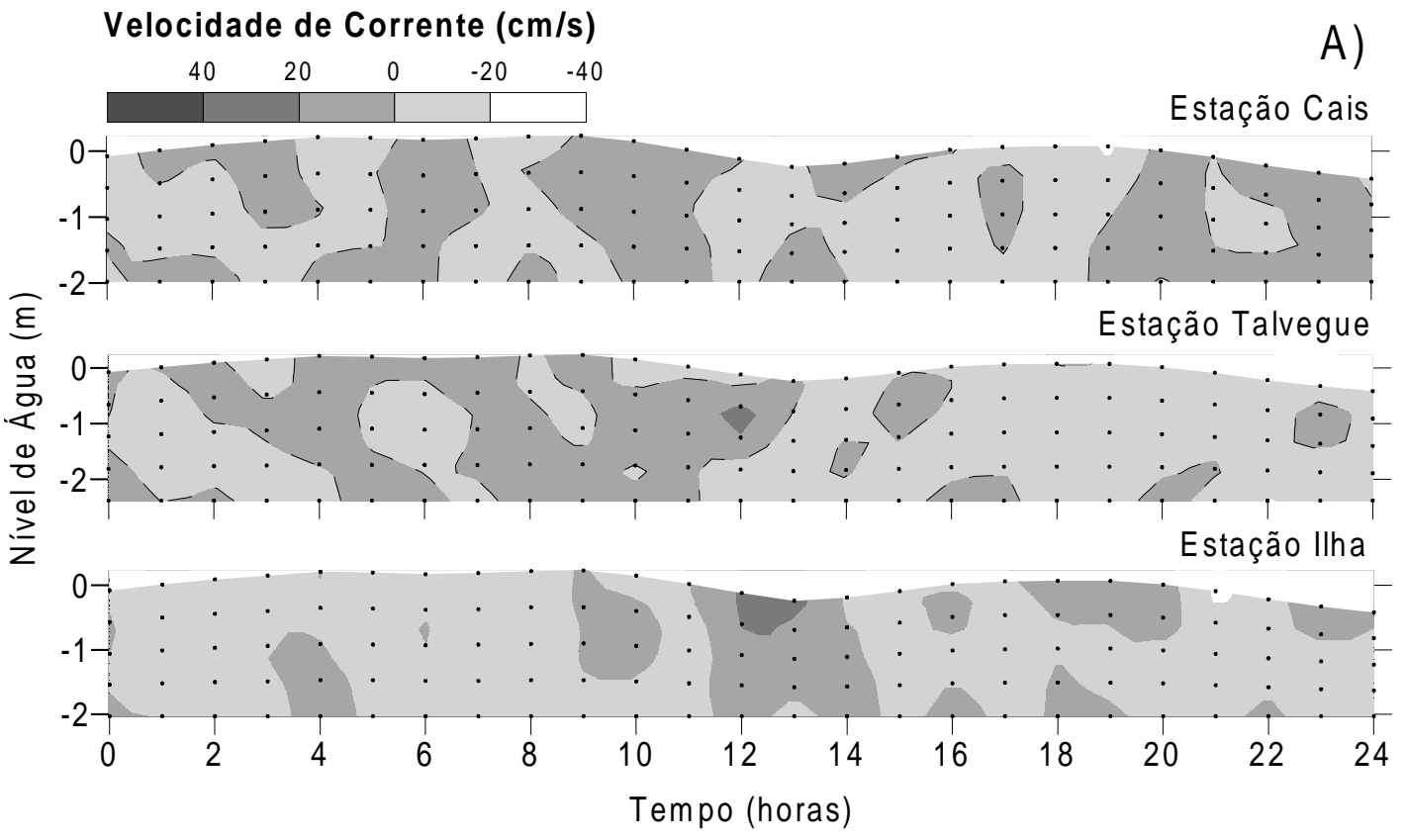

\section{Salinidade (PSU)}

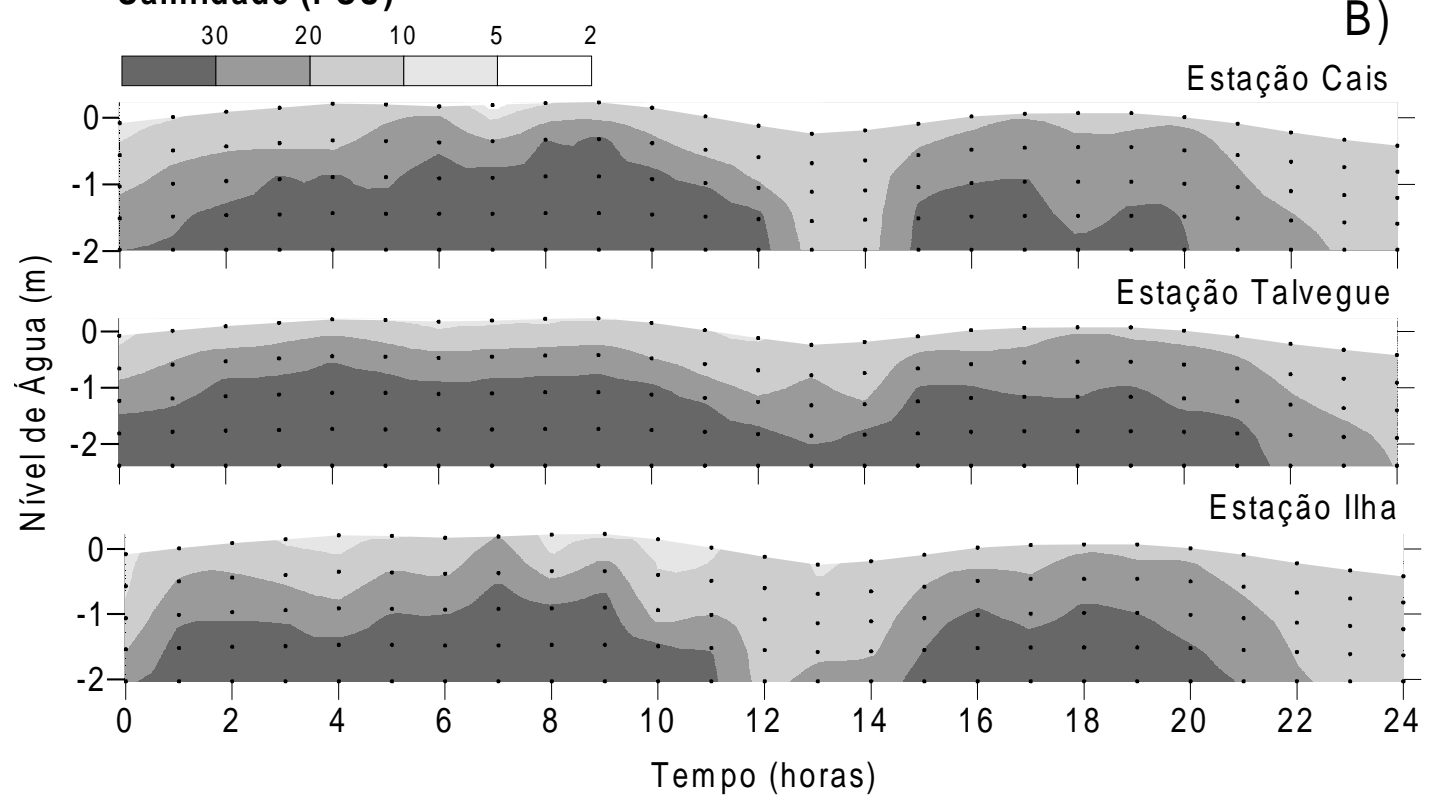

Figura 3 - Distribuição do campo de velocidade (A) e salinidade (B) durante período de maré de quadratura no estuário do Rio Camboriú. 
circulação, relativos as estações amostrais em cada período estudado, não apresentam uma variação significativa, isto é, não ocorre mudança na classificação entre os pontos amostrais.

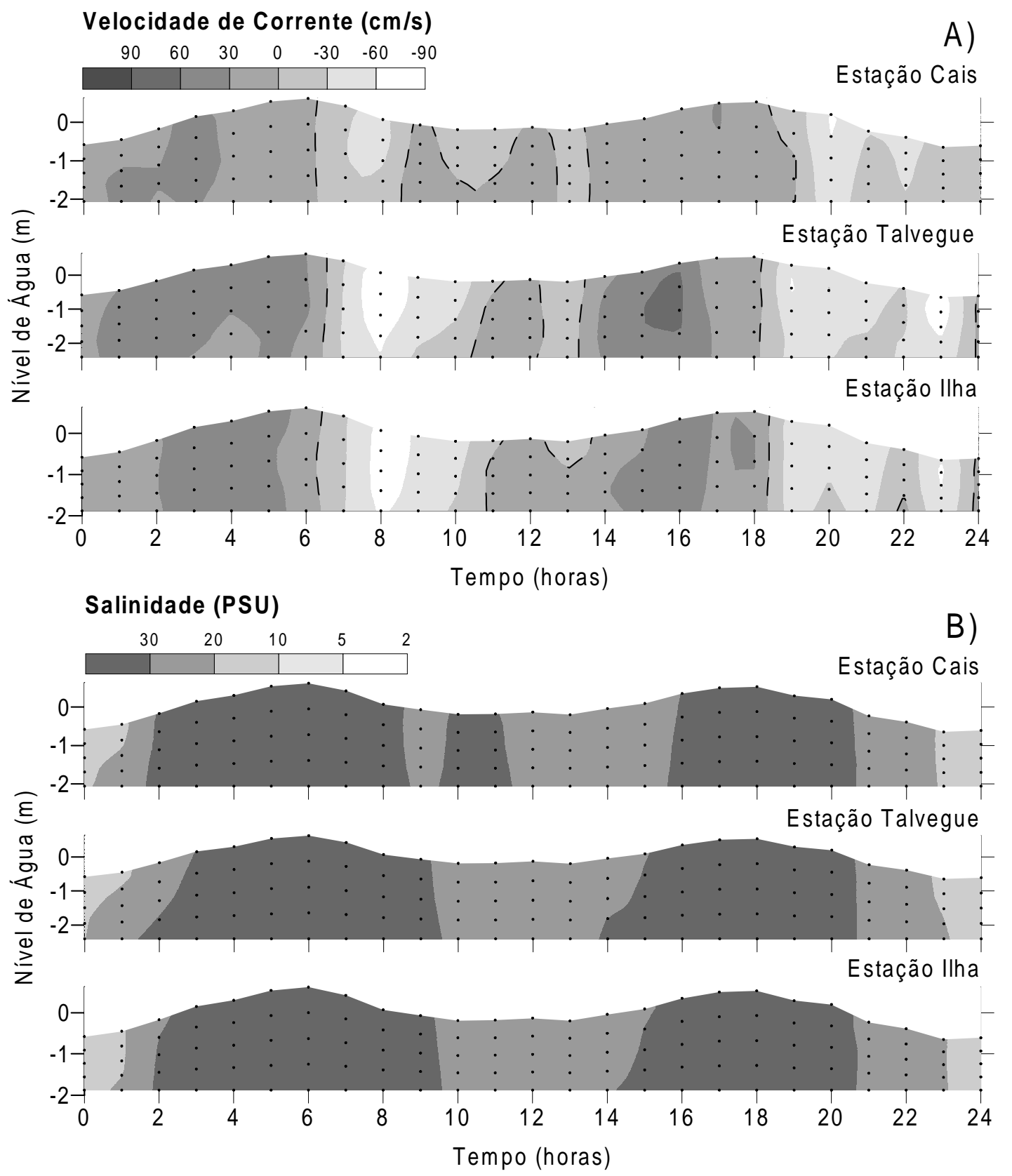

Figura 4 - Distribuição do campo de velocidade (A) e salinidade (B) durante período de maré de sizígia no estuário do Rio Camboriú. 

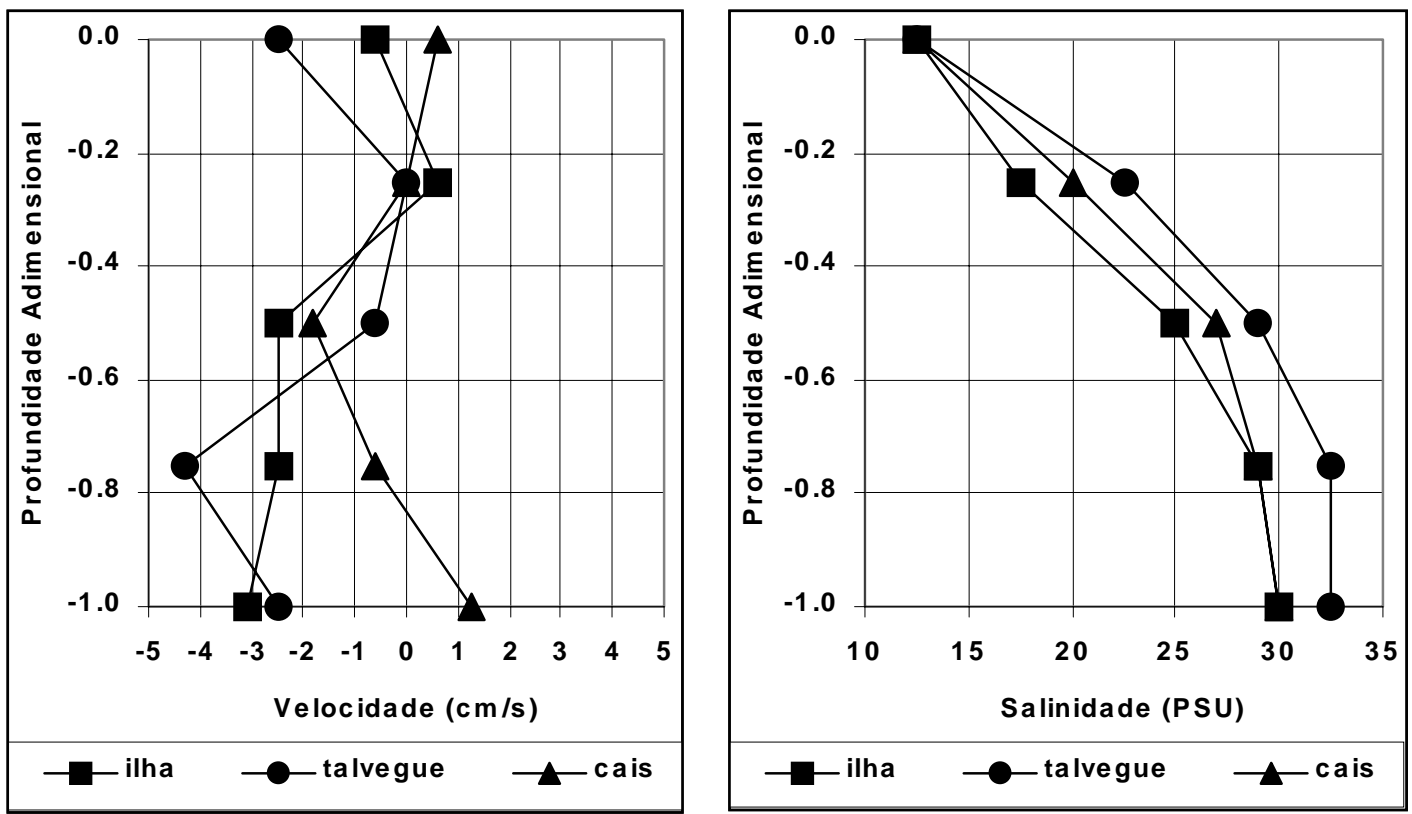

Figura 5 - Médias temporais de velocidade de corrente e salinidade para condições de maré de quadratura.
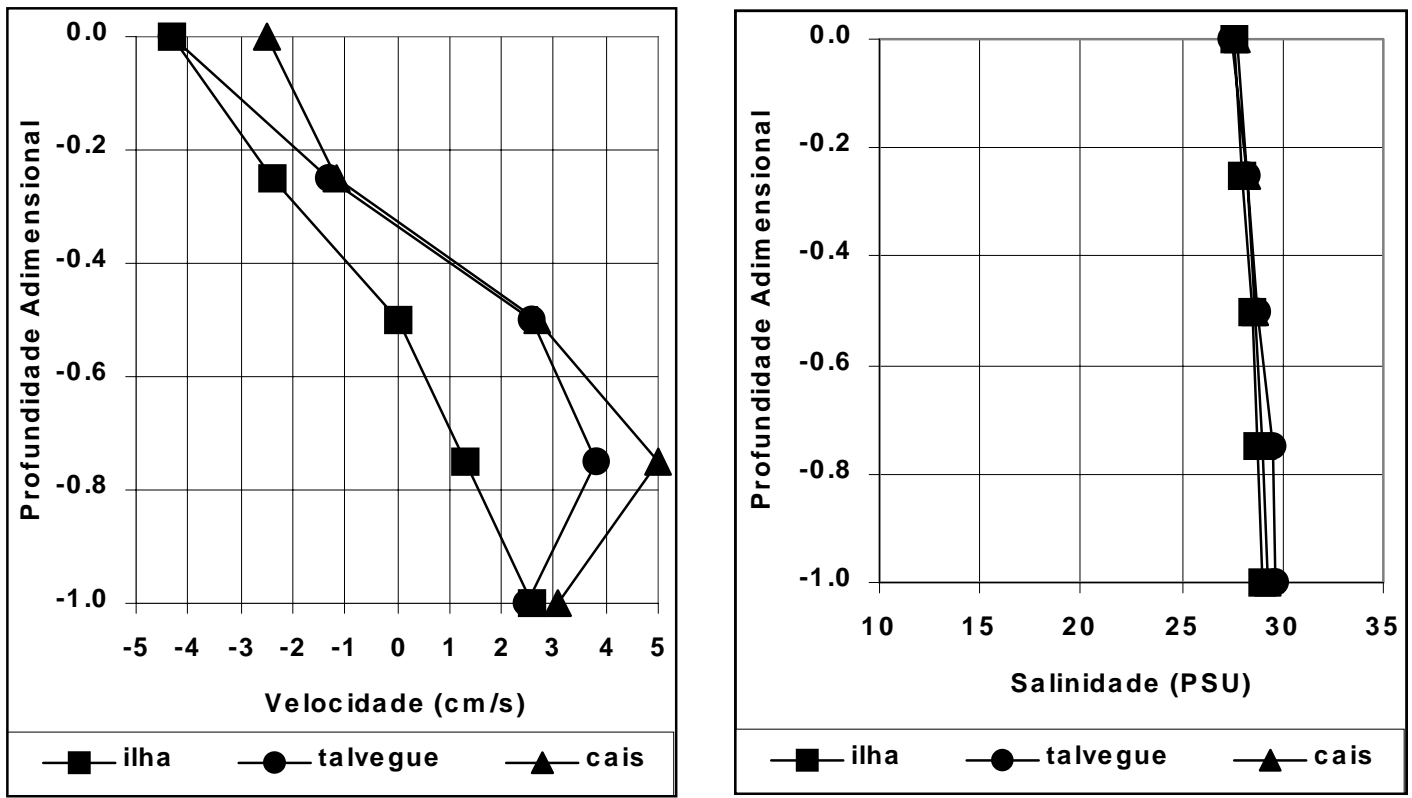

Figura 6: Médias temporais de velocidade de corrente e salinidade para condições de maré de sizígia. 
Para o período de maré de quadratura, os resultados indicaram que o transporte advectivo devido à descarga fluvial ( $1^{\circ}$ termo) é o principal mecanismo de transporte de sal neste estuário. Durante o período de maré de sizígia o principal mecanismo de transporte está associado a efeitos da maré ( $3^{\circ}$ termo). Os resultados obtidos, relativos a cada estação e período, são apresentados nas tabelas 1, 2 e 3.

O transporte total de sal é em direção ao continente durante a maré de quadratura e em direção ao oceano em maré de sizígia. É importante salientar que ocorre uma grande diferença nos valores obtidos entre as duas situações, sendo que em períodos de maré de sizígia o transporte total de sal pode ser até $90 \%$ superior ao observado durante a quadratura.

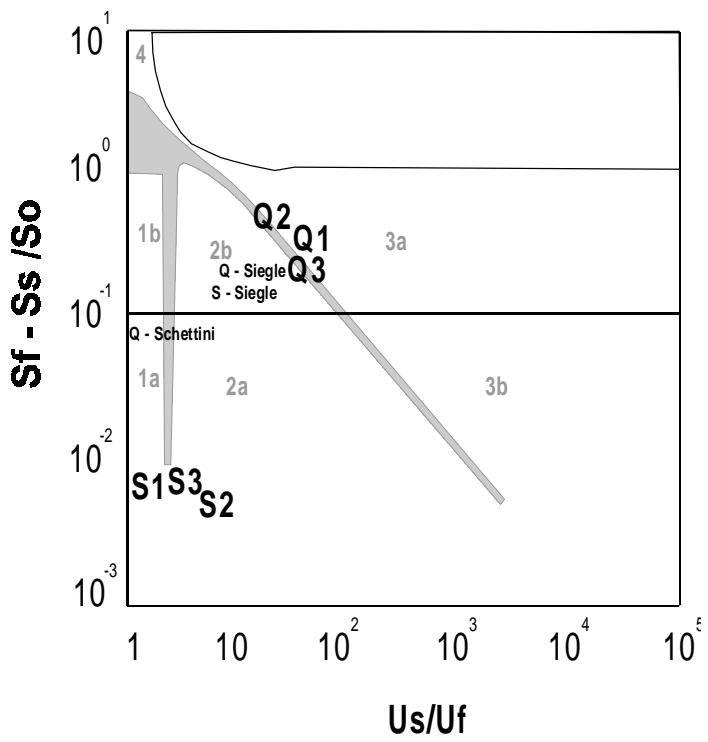

Figura 7 - Classificação do estuário do rio Camboriú em períodos de maré de quadratura (Q) e sizígia (S) em cada ponto amostral, sendo Ilha (1), talvegue (2) e Cais (3), de acordo com o diagrama de estratificaçãocirculação de Hansen \& Rattray (1966). Os resultados obtidos por Siegle (1999) e Schettini et al. (1996) em campanhas passadas estão representados no gráfico.

\section{DISCUSSÃO}

O gradiente vertical de salinidade do estuário do rio Camboriú varia em função da amplitude de maré. Com a descarga de água doce pela superfície ocorre a entrada de água salgada pelo fundo por advecção, proporcionando uma estratificação vertical durante o ciclo de maré. Durante o período de maré de sizígia, o efeito da amplitude de maré associado à baixa profundidade do sistema, proporciona uma coluna de água homogênea devido aos processos de difusão turbulenta presentes nestas condições.

Durante a maré de quadratura não é possível visualizar claramente a resposta da velocidade de corrente em relação à variação do nível de água. Segundo Siegle (1999), isto ocorre provavelmente devido à presença de constituintes harmônicas de águas rasas, formadas quando a maré progride sobre a plataforma continental adjacente. Durante a maré de sizígia fica evidente a resposta da velocidade de corrente em relação ao nível de água, pois neste período as constituintes semidiurnas estão em fase com as constituintes diurnas.

Durante a maré enchente e vazante, a água que entra ou sai do estuário possui maior velocidade no centro do canal devido os processos de fricção gerados nas bordas deste sistema, fazendo com que a velocidade diminua nestas áreas.

Em condições de maré de quadratura o coeficiente de estratificação é apenas 18\% maior $(0,89)$ que os valores encontrados nas margens e durante a maré de sizígia este coeficiente é cerca de $28 \%$ maior $(0,07)$.

No talvegue do canal o parâmetro de estratificação variou de 0,90 a 0,08 durante a maré de quadratura e sizígia, respectivamente. O parâmetro de circulação variou de 52 na maré de quadratura para 4 na maré de sizígia. A diferença entre estes parâmetros resultou na variação vertical da posição do estuário no diagrama, refletindo a intensificação da estratificação durante a maré de quadratura. 
Tabela 1 - Principais mecanismos de transporte no estuário do rio Camboriú - Ilha.

\begin{tabular}{cccccccc}
\hline Maré & Transporte Total & 10 & $2^{\circ}$ & $3^{\circ}$ & 40 & 50 & $6^{\circ}$ \\
\hline Quadratura & 21.61 & $\mathbf{1 5 . 5 0}$ & 0.00 & 0.00 & 0.00 & 4.31 & 1.80 \\
Sizígia & -75.11 & 23.07 & -1.76 & -6974 & 0.74 & -22.52 & -4.90 \\
\hline \hline
\end{tabular}

Tabela 2 - Principais mecanismos de transporte no estuário do rio Camboriú - Talvegue.

\begin{tabular}{cccccccc}
\hline \hline Maré & Transporte Total & 10 & $2^{\circ}$ & $3^{\circ}$ & 40 & 50 & $6^{\circ}$ \\
\hline Quadratura & 20.94 & $\mathbf{2 0 . 5 2}$ & 0.00 & 0.00 & 0.00 & -2.32 & 2.74 \\
Sizígia & -185.56 & -36.49 & 1.25 & -93.59 & -0.89 & -49.21 & -6.62 \\
*Quadrtura & 18.23 & $\mathbf{2 7 . 8 9}$ & -0.15 & -3.68 & 0.44 & -2.66 & -3.60 \\
* Sizígia & 43.68 & $\mathbf{3 1 . 0 3}$ & 0.67 & 4.45 & 1.24 & 0.29 & 5.98 \\
\hline \hline
\end{tabular}

Tabela 3 - Principais mecanismos de transporte no estuário do rio Camboriú - Cais.

\begin{tabular}{cccccccc}
\hline \hline Maré & Transporte Total & 10 & 20 & $3^{\circ}$ & 40 & 50 & $6^{\circ}$ \\
\hline Quadratura & 3.42 & $\mathbf{1 . 9 2}$ & 0.00 & 0.00 & 0.00 & 1.74 & -0.25 \\
Sizígia & -170.08 & -91.28 & 2.56 & -54.36 & -2.45 & -21.34 & -3.30 \\
\hline \hline
\end{tabular}

Durante condições de maré de sizígia, a seção transversal pequena (90 metros) e as baixas profundidades (cerca de 2 metros) associadas a uma alta amplitude de maré (1.2 metros), produziram processos de difusão turbulenta suficientemente intensos para uma completa quebra da haloclina, através do cisalhamento das correntes com o fundo. Segundo Dyer (1997), quando não há gradientes verticais de salinidade o fluxo vertical de sal é desprezível e o processo de mistura ocorre na direção longitudinal.

Durante maré de quadratura, é provável que características morfológicas associadas à presença de uma onda estacionária (seiche), originada da enseada de Balneário Camboriú, promovam uma diferenciação nos perfis de velocidade e direção de corrente entre as estações amostrais (Figura 3a). Através da aplicação da fórmula de Merian (in Pugh, 1987) estima-se um período natural de oscilação do seiche de cerca de 19 minutos. O período deste fenômeno depende das dimensões horizontais do ambiente estudado e da profundidade da água. Como a oscilação desta onda estacionária é mais freqüente que a onda de maré, uma das hipóteses para a diferença lateral das correntes, é que o tempo de amostragem entre uma estação e outra é suficientemente grande para que ocorra a ação do seiche antes desta ser realizada. Assim, para estas condições o método amostral referente aos dados de corrente não foi eficiente.

Durante maré de sizígia, a maior amplitude da maré possibilitou a ocorrência de velocidades maiores dentro do estuário, 
proporcionando uma maior correlação lateral entre as correntes (Figura 4a) e, portanto, a distribuição do campo de velocidade não sofreu ação da onda estacionária.

\section{CONCLUSÃO}

Durante maré de quadratura este estuário é classificado como parcialmente estratificado (Tipo $2 b$ ) e durante maré de sizígia como verticalmente homogêneo (Tipo 1a). Porém, não ocorre mudança na classificação entre os pontos amostrais. O sistema estudado apresenta uma variabilidade lateral de salinidade pequena.

Para o período de maré de quadratura, o principal mecanismo de transporte atuante no estuário é advectivo devido à descarga fluvial e, durante maré de sizígia, está associado a efeitos da maré. O transporte total de sal é em direção ao continente durante a maré de quadratura e em direção ao oceano em maré de sizígia.

A variação das velocidades de corrente não são concordantes com as oscilações do nível de água durante a maré de quadratura. Para a maré de sizígia está resposta é clara.

\section{AGRADECIMENTOS}

Os autores agradecem a Empresa de Pesquisa Agropecuária de Santa Catarina (EPAGRI) pelo fornecimento dos dados meteorológicos da região e a NAUTITUR por fornecer suas instalações durante realização das campanhas oceanográficas.

\section{REFERÊNCIAS BIBLIOGRÁFICAS}

Dyer, K.R. 1997. Estuaries: A Physical Introduction. $2^{\text {nd }}$ Ed. New York. John Wiley \& Sons.

Franklin-Silva, L. \& C.A.F. Schettini. 1997. Contribuição à hidrologia do estuário do Rio
Camboriú: avaliação da contribuição fluvial. X Semana Nacional de Oceanografia, Itajaí, Resumos Expandidos...FACIMAR/ UNIVALI. p.496-498.

Franklin-Silva, L.; Schettini, C.A.F. \& E. Siegle. 1998. Variabilidade lateral da salinidade no estuário do rio Camboriú. XI Semana Nacional de Oceanografia, Rio Grande, Resumos expandidos. p.419-421.

Hansen, D.V. \& M. Rattray Jr. 1966. New dimensions on estuarine classification. Limnology and Oceanography. p.319-326. Hunkins, K. 1981. Salt dispersion in the Hudson Estuary. Journal of Physical Oceanography. p. 729-738.

Kjerfve, B. 1979. Measurement and Analysis of Water Current, Temperature, Salinity, and Density. In: Dyer, K.R. Estuarine Hydrography and sedimentation - A handbook. London, Cambridge University Press. p186-226.

Kjerfve, B. 1986. Circulation and salt flux in a well mixed estuary. In: Van De Kreek, J. (ed.) Physics of shallow estuaries and bays. New York, Springer Verlag, p.22-29.

Miranda, L.B.; Castro, B.M. \& B. Kjerfve. 2002. Princípios de Oceanografia Física de Estuários. Edusp. São Paulo.

Pereira Filho, J.; Schettini, C. A.; Rorig, L. \& E. Siegle. 2001. Intratidal variation and net transport of dissolved inorganic nutrients, POC and chlorophyll a in the Camboriú River Estuary, Brazil. Estuarine, Coastal and Shelf Science. 53: 249-257.

Polette, M. 1999. Programa de orientação e mobilização parcipativa para a bacia hidrográfica do rio Camboriú subbacias do rio dos Macacos, rio Pequeno/Ostras e rio do Braço, Município do Camboriú (Santa Catarina). Documento Interno Univali.

Pugh, D.T. 1997. Tides, Surges and Mean SeaLevel - A Handbook For Engineers And Scientists. 2nd Ed. New York. John Wiley \& Sons.

Schettini, C.A.F.; Carvalho, J.L.B. \& P.J. Jabor. 1996. Hidrologia e distribuição de material em suspensão de quatro estuários de 
Santa Catarina. Simpósio Sobre Oceanografia, 3, São Paulo, Resumos... IO-USP. p.333.

Shubel, J.R. \& H.H. Carter. 1984. The estuary as a filter for fine-grained suspended sediment. In: KENNEDY, V.S. (ed.) The estuary as a filter. New York, Academic Press. p.81-105.

Siegle, E.; Klein, A.H.F.; Toldo Jr., E.E. \& C.A.F. Schettini. 1997. Análise dos dados pretéritos da desembocadura do rio Camboriú, Balneário Camboriú, SC. Anais, XII Simpósio Brasileiro de Recursos Hídricos. Vitória, ES. p.603-610
Siegle, E. 1999. Morfodinâmica da desembocadura do rio Camboriú, Balneário Camboriú - SC. Dissertação de Mestrado. UFRGS, Porto Alegre-RS.

Silva, A.Z.; Rorig, L. \& C. Resgalla Jr. 1999. Determinação do Efeito do Afluente da Estação de Tratamento de Esgotos de Balneário Camboriú Sobre o Crescimento de Skeletonema costatum. VII Congresso Brasileiro de Limnologia. Florianópolis, SC. Resumos. p.539. 